\title{
Tratamento da fratura-luxação de Lisfranc: relato de caso
}

Treatment of Lisfranc fracture-luxation: case report

Tratamiento de la fractura-luxación de Lisfranc: reporte de caso

Gustavo Pazoto NAKAMURA ${ }^{1}$

Amanda Oliva SPAZIANI ${ }^{2}$

Talita Costa BARBOSA2

Beatriz Silva FERRARI 2

Raissa Silva FROTA ${ }^{3}$

Taís Cristina Nascimento MARQUES ${ }^{4}$

José Antonio FRANCISCON ${ }^{5}$

Daniel Brito FERNANDES ${ }^{5}$

Flávio Henrique Nuevo Benez dos SANTOS ${ }^{5}$

${ }^{1}$ Médico residente em Ortopedia e Traumatologia no Hospital Ensino Santa Casa de Misericórdia de Fernandópolis, $15601-012$ Fernandópolis-SP, Brasil

${ }^{2}$ Graduanda em Medicina, Universidade Brasil - Campus Fernandópolis, 15600.000 Fernandópolis-SP, Brasil

${ }^{3}$ Graduanda em Medicina, Universidade de Rio Verde - Goianésia (GO) 76380-000

${ }^{4}$ Docente de medicina, Universidade Brasil - Campus Fernandópolis, 15600.000 Fernandópolis-SP, Brasil

${ }^{5}$ Médico Ortopedista e Traumatologista no Hospital Ensino Santa Casa de Misericórdia de Fernandópolis 15601-012 Fernandópolis-SP, Brasil

\section{Resumo}

A fratura-luxação da articulação tarsometatársica é rara, tendo incidência em torno de 1 para 55.000 pessoas por ano, recebe o nome Lisfranc em homenagem ao médico que descreveu uma amputação desta articulação. Considerando isso, esse estudo visa relatar um caso de fraturaluxação de Lisfranc e seu tratamento em um paciente sexo masculino, 21 anos, sem comorbidades. Vítima de acidente motociclistico; trazido pelo SAMU com imobilização por talas em pé direito, em bom estado geral e Glasgow 15. Exame físico do pé direito: dor, edema em região dorsal, pele íntegra, pulsos presentes, sem alterações neurológicas ou vasculares. Após raio-x e tomografia computadorizada do pé, foi diagnosticado com fratura-luxação de Lisfranc. Nove dias após, foi submetido a osteossíntese com passagem de fio de kirschnner em base da falange proximal do hálux e redução e fixação da cabeça do segundo metatarso. Passagem de 2 parafusos corticais do cubóide para base do segundo metatarso e do terceiro para a cuneiforme medial e imobilização com tala em pé. Paciente evoluiu bem e seguiu com alta hospitalar e seguimento ambulatorial. A fratura-luxação de Lisfranc é uma lesão grave que apresenta complicações incapacitantes, sendo a mais importante delas a osteoartrose pós-traumática, que pode evoluir com dor e limitação funcional. É importante manter a qualidade da redução, assim como o tratamento cirúrgico precoce visa prevenir a síndrome compartimental do pé, complicação aguda mais frequente.

Descritores: Fraturas Ósseas; Fixação Interna de Fraturas; Pé.

\section{Abstract}

The fracture-dislocation of the tarsometatarsal joint is rare, with an incidence of around 1 to 55,000 people per year. It is called Lisfranc in honor of the physician who described an amputation of this joint. Considering this, this study aims to report a case of Lisfranc fracturedislocation and its treatment in a male patient, 21 years old, without comorbidities. Victim of motorcycle accident brought by SAMU (Emergency Mobile Care Service) with immobilization by splints in the right foot in good general condition and Glasgow 15 . Physical examination of the right foot determined: pain, edema in the dorsal region, whole skin, present pulses, without neurological or vascular alterations. After applying X-ray and computed tomography of the foot, he was diagnosed with fracture-dislocation of Lisfranc. Nine days later, he was submitted to an osteosynthesis with wire passing of kirschner at the base of the proximal hallux phalange and reduction and fixation of the second metatarsal head. The passage was made with 2 cortical screws from the cuboid to the base of the second metatarsal. A third screw was applied at the medial cuneiform and immobilization was made with standing splint. Patient progressed well and he was discharged from hospital followed up with and outpatient procedure. Lisfranc fracture-dislocation is a severe lesion with disabling complications, the most important being post-traumatic osteoarthrosis, which may progress with pain and functional limitation. It is important to maintain the quality of the reduction, just as early surgical treatment which aims to prevent the compartmental foot syndrome, a more frequent acute complication.

Descriptors: Fractures Bone; Fracture Fixation, Internal; Foot.

\section{Resumen}

La fractura-luxación de la articulación tarsometatarsiana es rara, con una incidencia de alrededor de 1 a 55,000 personas por año. Se 1lama Lisfranc en honor del médico que describió una amputación de esta articulación. Teniendo en cuenta esto, este estudio pretende informar un caso de fractura-luxación de Lisfranc y su tratamiento en un paciente masculino, de 21 años, sin comorbilidades. Víctima de accidente de motocicleta; fue traído por el Servicio de Atención Móvil de Urgencia (SAMU) con inmovilización por férulas en pie derecho, en buen estado general y Glasgow 15. Fue realizado un examen físico en el pie derecho, el cual determinó: dolor, edema en la región dorsal, piel entera, pulsos presentes, sin alteraciones neurológicas ni vasculares. Después de haber realizado una radiografía y tomografía computarizada del pie, se le diagnosticó fractura-luxación de Lisfranc. Nueve días más tarde, se sometió a una osteosíntesis con el paso de alambre de kirschner en la base de la falange del hallux proximal y la reducción y fijación de la segunda cabeza metatarsiana. Fueron implantados 2 tornillos corticales desde el cuboides a la base del segundo metatarsiano y el tercero a la cuneiforme medial con una posterior inmovilización con férula en el pie. El paciente progresó bien, por lo cual fue dado de alta y seguimiento ambulatorio. La fractura-luxación de Lisfranc es una lesión grave con complicaciones incapacitantes, la más importante es la artrosis postraumática, que puede intensificarse con el dolor y la limitación funcional. Es importante mantener la calidad de la reducción, al igual que el tratamiento quirúrgico temprano, que tiene como objetivo prevenir el síndrome compartimental del pie, complicación aguda más frecuente..

Descriptores: Fractura Óseas; Fijación Interna de Fracturas; Pie.

\section{INTRODUÇÃO}

A fratura-luxação da articulação

tarsometatársica é rara, possui incidência em torno de 1 para 55.000 pessoas por ano e recebe o nome Lisfranc em homenagem ao médico que descreveu uma amputação desta articulação ${ }^{1}$.

Relatos de incidência dessa modalidade de fratura induzem que ela seja-por trauma de alta energia, tem como característica especial a dificuldade de ser diagnostica imediatamente, os números mostram que cerca de $2 \%$ do total de fraturas são dessa espécie de traumatismo. Ressaltase que em politraumatizados, necessitam de uma 
atenção maior no estado geral e em lesões mais alarmantes do que a presente no pé ${ }^{1,2}$.

Este tipo de lesão constantemente evolui acompanhada da síndrome compartimental e como há dificuldade de ser diagnosticada ou a situação passa desapercebida os dedos dos pés podem apresentar deformidade expressiva ${ }^{3,4}$.

O tratamento terapêutico fundamenta-se preferencialmente em procedimento cirúrgico. Basicamente concentra-se na redução anatômica e fixação estável. O tratamento cirúrgico é utilizado pelos profissionais da saúde com maior frequência em razão dos diferentes estudos indicarem maior sucesso nessa modalidade de tratamento quando comparada com tratamento conservador. Os estudos mostram que os resultados obtidos nos tratamentos conservadores não se revelam satisfatórios ${ }^{3}$.

Em alguns casos foi observado que o tratamento de primeira escolha do tipo cirúrgico não é totalmente satisfatório, pois o paciente não apresenta a evolução esperada e como uma opção de proteção e segurança recorre-se a técnica de artrodese, que, basicamente, caracteriza-se pela fixação cirúrgica de uma determinada articulação ${ }^{5}$.

A escolha do tipo de tratamento tem variações significativas, mas tomada como base a revisão realizada pelo Projeto Diretrizes ${ }^{6}$, a lesão que se apresenta com perda de alinhamento com o pé, não tratada adequadamente, e aquela que não há espaço para o tratamento conservador, torna-se necessário a redução anatômica e a fixação estável ${ }^{7}$.

Os sintomas não são, em muitos casos, padronizados, porém, de forma geral, são: dor e edema na região dorsal do pé, dificuldade em apoiar o pé afetado no solo e hematoma na região plantar do pé ${ }^{8}$.

Considerando isso, este estudo objetiva relatar um caso de fratura-luxação de Lisfranc e, respectivamente, o seu tratamento.

\section{CASO CLÍNICO}

Paciente do sexo masculino, 21 anos vítima de acidente motociclístico versus carro, foi trazido pelo serviço médico de urgência corretamente imobilizado, atendimento pelo médico generalista de plantão que descartou demais lesões, realizou analgesia e transferiu o paciente para equipe de Ortopedia e Traumatologia.

Ao exame físico do pé direito, apresentou dor a palpação e movimentação da articulação, edema em região dorsal, equimose plantar na região do médiopé, pele íntegra, pulsos presentes, impossibilidade de sustentar peso, ausência de alterações neurológicas ou vasculares.

Inicialmente realizaram-se testes de compressão lateral, estabilidade axial entre primeiro e segundo metatarso ou de pronação passiva com abdução do antepé com retropé, os resultados obtidos foram positivos.

No exame de imagem realizado constatou-se fratura de falange proximal do hálux e terceiro metatarsiano, luxação metatarso-falangiano do hálux e do segundo dedo e luxação metatarso-tarso do segundo, terceiro e quarto dedos. Com o auxílio da tomografia computadorizada do pé foi possível observar fratura do terço proximal da falange proximal do hálux, com discreto desvio superior do fragmento, há traço linear de fratura na epífise distal do terceiro metatarso, sem desalinhamento, luxação da articulação metatarsofalangiana do segundo dedo, estruturas ósseas e de partes moles do retropé íntegras e com densidade preservada.

Dessa forma o paciente foi diagnosticado com fratura-luxação de Lisfranc. Após nove dias, o paciente foi submetido a osteossíntese com passagem de fio de kirschnner em base da falange proximal do hálux e redução e fixação da cabeça do segundo metatarso. Passagem de dois parafusos corticais do cubóide para base do segundo metatarso e do terceiro para a cuneiforme medial e imobilização com tala em pé. Houve evolução significativa do paciente, que permitiu a alta hospitalar e, consequentemente, seguimento ambulatorial (Figuras 1 e 2).

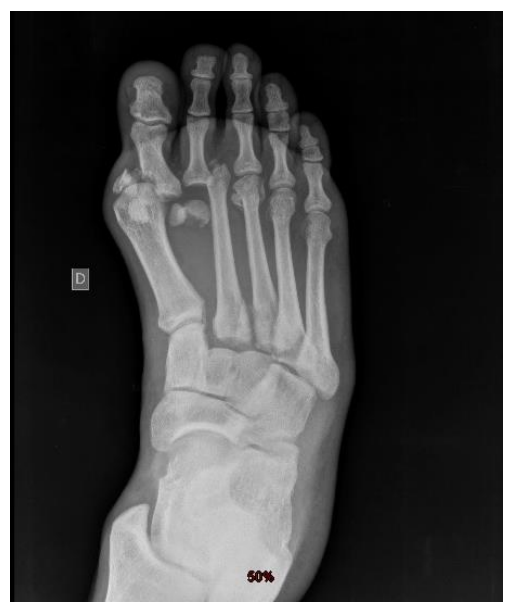

Figura 1: Pré-operatório

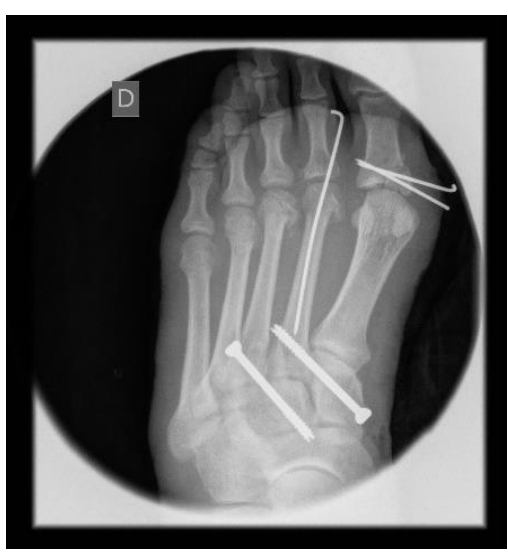

Figura 2: Pós-operatório

DISCUSSÃO

A fratura-luxação de Lisfranc tem a característica de ser lesão grave que pode apresentar complicações incapacitantes, sendo a mais 
importante delas a osteoartrose pós-traumática, que pode evoluir com dor e limitação funcional ${ }^{7}$.

Nota-se a importância de se manter a qualidade da redução, bem como o tratamento cirúrgico precoce, pois, os dois procedimentos têm como objetivo principal prevenir a síndrome compartimental do pé, relatada como complicação aguda mais frequente ${ }^{3}$.

É relevante um atendimento inicial completo, sem menosprezar regiões corporais, como o pé. Além de avaliação inicial correta é preciso estar atento às questões clínicas deste tipo de lesão, uma vez que têm características próprias e, como consequência positiva, pode livrar $o$ paciente de alguma incapacidade irreversível ${ }^{3}$.

Exame complementares, tais como - $\mathrm{X}$, tomografia computadorizada e mesmo ressonância magnética nuclear são estratégicos para confirmação do diagnóstico inicial. Se for realizado o exame radiográfico simples recomenda-se realizar o exame nas incidências AP, lateral e oblíqua média a $30^{\circ}$, incluindo o pé contra-lateral e associação com carga ou algum fator de estresse. Os demais exames servem para caracterizar mais especificamente lesões ósseas e ligamentares ${ }^{9}$.

Como ainda não há consenso no manejo deste tipo de lesão, é salutar analisar as necessidades do paciente, verificando qual dos tratamentos utilizados poderá trazer maior conforto e benefício para o mesmo?

Há necessidade, no tratamento conservador, que as lesões estejam estáveis. Nesse sentido, há relatos que mostram ganhos funcionais significativos quando adotado esse procedimento inicial. Entretanto, como a maioria dos casos tratam de lesões instáveis, recomenda-se, de imediato, o tratamento cirúrgico. Esse apresenta melhores resultados com a redução aberta e fixação interna, sendo a redução da base do segundo metatársico o passo fundamental da cirurgia. Outro recurso que pode ser empregado é o parafuso, utilizado como método de fixação das lesões que afetam as articulações tarsometatársicas das colunas medial e média, mas as placas dorsais, pelo menor dano que imposto à cartilagem articular, mostram-se ser teoricamente, vantajosas ${ }^{3}$.

A artrodese parcial primária mostra-se, nos estudos, benéfica nas lesões puramente ligamentares das colunas medial e média, com instabilidade multidirecional nas luxações das tarsometatársicas graves $^{10}$.

\section{CONCLUSÃO}

Conclui-se que a fratura-luxação de Lisfranc é uma lesão grave das articulações tarsometatarsais que pode gerar consequências graves agudas e crônicas ao paciente. $\mathrm{O}$ diagnóstico precoce associado à rápida redução anatômica e fixação estável proporcionam melhores resultados. O paciente jovem, referido no presente relato de caso, obteve resultados satisfatórios graças ao rápido diagnóstico e correta estabilização.

\section{REFERÊNCIAS}

1. Hebert SK, Barros Filho TEP, Xavier R, Pardini Júnior AG. Ortopedia e traumatologia: princípios e prática. 5. ed. São Paulo: Artmed; 2016.

2. Ly TV, Coetzee JC. Treatment of primarily ligamentous Lisfranc joint injuries: primary arthrodesis compared with open reduction and internal fixation. A prospective, randomized study. J Bone Joint Surg Am 2006;88(3):514-20.

3. Kuo RS, Tejwani NC, Digiovanni CW, Holt SK, Benirschke SK, Hansen ST Jr et al. Outcome after open reduction and internal fixation of Lisfranc joint injuries. J Bone Joint Surg Am 2000; 82(11):1609-18.

4. Garcia-Renedo RJ, Carranza-Bencano A, LealGomez R, Cámara-Arrigunaga F. Análisis de las complicaciones en pacientes con fractura-luxación de Lisfranc. Acta ortop mex. 2016;30(6):284-90.

5. 5-Sangeorzan BJ, Veith RG, Hansen ST Jr. Salvage of Lisfranc's tarsometatarsal joint by arthrodesis. Foot Ankle. 1990;10(4):193-200.

6. Pereira CJ, Canto RST, Tramontini JL, Canto FRT. Fratura-Luxação Tarsometatarsiana (Lisfranc). Projeto Diretrizes. Associação Médica Brasileira e Conselho Federal de Medicina. Sociedade Brasileira de Ortopedia e Traumatologia; 2007.

7. Pina-Vaz J. Fractura de Lisfranc: controvérsias do diagnóstico e tratamento [dissertação]. Porto: Faculdade de Medicina da Universidade do Porto; 2012.

8. Rodrigues CS. Patologia aguda da articulação de Lisfranc. Uma entidade complexa e frequentemente esquecida. Faculdade de Medicina da Universidade de Lisboa. Clínica Universitária de Ortopedia; 2016.

9. Velázquez-Vélez D, Durán-Martínez N, PeñafortGarcía JA, Romero-Peña A. Control de daño de extremidad pélvica en lesión compleja del pie: Reporte de un caso. Acta ortop mex. 2015;29(5):275-79.

10. Sobrado MF, Saito GH, Sakaki MH, Pontin PA, Santos ALG, Fernandes TD. Estudo epidemiológico sobre lesões lisfrancas. Acta ortop. bras. 2017;25(1):44-7.

\section{CONFLITO DE INTERESSES}

Os autores declaram não haver conflitos de interesse.

\section{AUTOR PARA CORRESPONDENCIA}

\section{Gustavo Pazoto Nakamura}

gustavo_pazoto@hotmail.com 